\title{
Abundância de microartrópodes do solo em fragmentos de mata com araucária no sul do Brasil
}

\author{
Marcelo Maisonette Duarte
}

\begin{abstract}
Seção de Conservação e Manejo, Museu de Ciências Naturais, Fundação Zoobotânica do Rio Grande do Sul, Rua Dr. Salvador França, 1427, 90690-000 Porto Alegre, RS, Brasil. (manejo@fzb.rs.gov.br)
\end{abstract}

\begin{abstract}
Abundance of soil microarthropods in araucaria forest fragments in southern Brazil. The abundance of soil microarthropods from seven fragments of Araucaria Forest, Muitos Capões, Rio Grande do Sul, Brazil, was compared. The size of the fragments ranged from 0.25 ha to 35 ha, the two largest fragments are situated within the Aracuri Ecological Station and the remaining five are situated in a cattle ranching farm. In June 2000, three plots $(10 \mathrm{~m} \times 10 \mathrm{~m})$ were established in the central area of each patch, and three soil cores $(7 \mathrm{~cm}$ diameter $\times 6$ $\mathrm{cm}$ deep) were taken per plot. The abundance of microarthropods in the upper six centimeters (soil + litter) varied between 63209 and 102704 ind.m $^{-2}$, with oribatid mites (Acari, Cryptostigmata) being dominant in all fragments (between $46.9 \%$ and $61.3 \%$ of total individuals). Most microarthropod groups presented a decrease in abundance with decreasing fragment area, with a statistically significant difference between smaller and larger fragments. The proportion of oribatids also decreased with decreasing fragment area. The results suggest that the growing fragmentation process of Araucaria forests in southern Brazil, associated to a tendency for reducing the size of remnant fragments, can affect the abundance of soil microarthropods, and therefore, the quality and health of this ecosystem.
\end{abstract}

KEYWORDS. Soil microarthropods, araucaria forest, Acari, Collembola, habitat fragmentation.

\section{INTRODUÇÃO}

Os microartrópodes do solo apresentam enorme abundância e diversidade em ecossistemas florestais do mundo todo (Petersen \& LuXTON, 1982; WALLWORK, 1970), sendo importantes para os processos de decomposição e mineralização da matéria orgânica (BUTCHER et al., 1971; SeAdSTed, 1984; Beare et al., 1995; Lavelle et al., 1995), e com isso influenciando os níveis locais de fertilidade e todo o funcionamento de muitos ecossistemas terrestres (WitKamp, 1971; Whitford, 1996; Heneghan \& Bolger, 1998). Maraun et al. (1998) sugerem ser os microartrópodes que se alimentam de fungos do solo importantes para a resiliência de sistemas florestais, por promoverem, após algum distúrbio, uma rápida recuperação da biomassa microbiana, a recolonização da serapilheira pelos fungos e a estabilização dos nutrientes. Apesar disso, os organismos do solo têm tido uma influência insignificante no desenvolvimento da teoria ecológica contemporânea (WARDLE \& GILLER, 1996), com uma exceção podendo ser feita com relação à teoria dos corredores ecológicos em paisagens fragmentadas (Gilbert et al., 1998; Gonzalez \& Chaneton, 2002). Os microartrópodes podem ser indicadores valiosos dos impactos antropogênicos em florestas (VAN STRAALEN $e t$ al., 1988; Cancela da FonseCA, 1990; Rusek \& Marshall, 2000; Donegan et al., 2001), assim como em diversos agroecossistemas (Mueller et al., 1990; WARDLE et al., 1995; Behan-Pelletier, 1999).

No Brasil, são escassos os estudos sobre microartrópodes do solo (Adis et al., 1987; VALLEJo et al., 1987; Ribeiro \& SchUBART, 1989; HARADA \& BANDEIRA, 1994a,b; Ushiwata et al., 1995; Franklin et al., 1998; DuARTE \& BeCKer, 2000; CuliK et al., 2002). Esta situação é contrastante com a enorme velocidade com que extensas áreas de terra vêm sendo transformadas em áreas degradadas. A crescente fragmentação dos ecossistemas florestais é um dos grandes problemas ambientais do mundo moderno (SAUNDERS et al., 1991; LAURANCE et al., 2002). Entre os diversos ecossistemas ameaçados encontram-se as florestas com Araucaria angustifolia (Bertol.) O. Ktze. no sul do Brasil (Floresta Ombrófila Mista).

A vegetação do sul do Brasil no período précolombiano pode ser descrita como um mosaico de campos e matas com araucária (A. angustifolia) nos planaltos, florestas tropicais úmidas nos vales e planícies (BeHLing, 1997). A mata com araucária ocupava, originalmente, uma área de cerca de $181.795 \mathrm{~km}^{2}$ (SILVA, 1996), restando atualmente algo em torno de $21.213 \mathrm{~km}^{2}$ (BRASIL, 1993), valor de difícil determinação, uma vez que a ação antrópica exercida desde o início da colonização desta região descaracterizou seus limites originais, com os fragmentos remanescentes encontrando-se atualmente em diferentes estágios de degradação ambiental (Silva, 1996; Behling, 1997).

Apesar da grande diversidade de animais (CABRERA \& WilLINK, 1980), estudos ecológicos sobre a fauna associada a este ecossistema florestal tipicamente sulbrasileiro são escassos e, para os microartrópodes do solo, praticamente inexistentes. Objetiva-se investigar a abundância de microartrópodes do solo em sete fragmentos de mata com araucária de diferentes tamanhos e sob diferentes pressões antrópicas no sul do Brasil.

\section{MATERIAL E MÉTODOS}

O estudo foi realizado em sete fragmentos de mata com araucária localizados no município de Muitos Capões (28 $8^{\circ} 3^{\prime}$ S; 5 $1^{\circ} 10^{\prime} \mathrm{W}$ ), Rio Grande do Sul, Brasil. 
Duas destas áreas (A1 e A2) encontram-se na Estação Ecológica de Aracuri (ESEC) e outras cinco (M1, M2, M3, M4 e M5) pertencem à fazenda Boa Vista, localizada cerca de $10 \mathrm{~km}$ ao sul da ESEC, cuja principal atividade é a pecuária de corte.

$\mathrm{O}$ fragmento M1, com 6 ha, representa uma mata ciliar do rio Saltinho, tributário da bacia Taquari-Antas. Este fragmento sofre ocasionalmente um pulso de inundação que afeta a camada de serapilheira (cerca de $1,0 \mathrm{~cm}$ na época da amostragem). O fragmento M2, com cerca de 4 ha, situa-se na nascente de um pequeno riacho, que corre para o rio Saltinho. Os fragmentos M1 e M2 são conectados por um estreito corredor de mato e um banhado, porém o pulso de inundação ocasional não atinge M2, o qual tem uma camada de serapilheira entre $1-2 \mathrm{~cm}$. O fragmento $\mathrm{M} 3$ tem cerca de 0,8 ha, apresentando áreas de intenso pisoteio pelo gado onde a serapilheira é escassa $(<1 \mathrm{~cm})$. O fragmento M4, com cerca de 0,4 ha, é o mais aberto e com estrato arbóreo alterado, tendo também serapilheira mais escassa $(<0,5 \mathrm{~cm})$. O fragmento M5 é o menor dentre os amostrados; com cerca de 0,25 ha, apresenta uma camada de serapilheira mais espessa que M3 e M4, entre $1-2 \mathrm{~cm}$.

$\mathrm{O}$ fragmento A1 tem cerca de 18 ha, sendo adjacente à sede da ESEC, antiga sede da fazenda. A área do fragmento A2 é maior, com aproximadamente 35 ha, estando conectado ao A1 por uma faixa de mata ciliar de um pequeno riacho, afluente do rio Santa Rita, também tributário da bacia Taquari-Antas. Ambos possuem espessa camada de serapilheira $(1-3 \mathrm{~cm})$, apresentando porém diferenças quanto à densidade de araucárias, nitidamente mais alta em A2.

As diferentes formações vegetais existentes na ESEC foram estudadas por WAECHTER et al. (1984), Cestaro et al. (1986), Longhi-Wagner \& Boldrini (1988) e Fleig (1990) e podem ser consideradas características dos fragmentos de mata com araucária da região de Muitos Capões. Os sete capões de mato estudados sofreram ao longo das últimas décadas diversas alterações antropogênicas, em função das atividades desenvolvidas nas fazendas (pecuária, lavouras), bem como pela retirada de lenha e madeiras nobres. Os fragmentos A1 e A2, além da maior área remanescente, tiveram estes impactos estancados com a criação da ESEC em 1980 (CESTARO et al., 1986).

A amostragem foi realizada nos dias 3 e 4 de junho de 2000. Em cada um dos sete fragmentos, três parcelas contíguas de $10 \mathrm{~m}$ x $10 \mathrm{~m}$ foram demarcadas na área mais central possível. Em cada parcela, três unidades amostrais de $7 \mathrm{~cm}$ de diâmetro por $6 \mathrm{~cm}$ de profundidade (serapilheira + solo) foram retiradas aleatoriamente. Cada amostra foi colocada em saco plástico individual e mantida em caixa térmica durante o transporte até o laboratório de Ecologia Animal, Universidade Federal do Rio Grande do Sul, onde as amostras foram processadas durante sete dias em extratores tipo Berlese-Tullgren modificados (DUARTE \& BECKER, 2000). Após a extração, os microartrópodes foram exaustivamente triados sob estereomicroscópio, sendo classificados até o nível de ordem. Todos os organismos amostrados encontram-se depositados nas coleções do Museu de Ciências Naturais, Fundação Zoobotânica do Rio Grande do Sul, Porto Alegre. Para a classificação dos diferentes grupos utilizou-se: BAKER \& Wharton (1959), Balogh (1972), Balogh \& Balogh (1988, 1990), Dindal (1990) e Eisenbeis \& Whichard (1987).

Para a análise estatística, os microartrópodes foram subdivididos em quatro grupos: ácaros oribatídeos; outros ácaros; colêmbolos; outros artrópodes. Foi aplicada aos dados, com o objetivo de verificar possíveis diferenças na abundância destes quatro grupos entre os sete fragmentos, uma análise multivariada utilizando-se o programa MULTIV (versão 1.2. Copyright (C Pillar, V. de P., Dep. Ecologia/UFRGS, Porto Alegre, 1998). Nesta análise utilizou-se o número médio de indivíduos dos quatro grupos definidos em cada uma das três parcelas de cada fragmento. Dentre as opções do MULTIV, dois testes foram aplicados: um teste de aleatorização e uma análise exploratória (ordenação, análise de coordenadas principais). Um detalhamento sobre os procedimentos destes testes pode ser encontrado em Pillar \& Orlóci (1996).

\section{RESULTADOS}

Com relação à densidade média (ind. $\mathrm{m}^{-2}$ ) e em termos percentuais (tab. I), o grupo dos ácaros oribatídeos foi amplamente dominante em todos os fragmentos, apresentando porém um percentual menor em M3 e M4, os quais estão entre os menores e são os mais impactados. A abundância de oribatídeos registrada em M1, M2 e M5 deve-se principalmente à superfamília Oppioidea, com Microppia cf. minus sendo amplamente dominante, chegando a representar até $80 \%$ de todos os indivíduos em uma das parcelas de M5. Outras espécies de oribatídeos comuns a todas as áreas foram Tectocepheus cf. velatus, Humerobates sp., Camisia segnis (Hermann, 1804) e Epilohmannia sp. Em A1 e A2, maiores e menos impactados, foi encontrada maior diversidade de ácaros oribatídeos, sendo que Charassobates sp. e Eremobelba sp. somente foram registradas em A2.

$O$ resultado do teste de aleatorização indicou diferenças significativas entre os fragmentos $(\mathrm{p}=$ 0,007). Os menores (M3, M4 e M5) não diferiram significativamente entre si, porém ocorreram diferenças significativas entre M4 (nitidamente o mais alterado) e os quatro maiores (M1, $\mathrm{p}=0,013 ; \mathrm{M} 2, \mathrm{p}=0,001 ; \mathrm{A} 1, \mathrm{p}$ $=0,005 ; \mathrm{A} 2, \mathrm{p}=0,007)$ e entre M3 e A $1(\mathrm{p}=0,034)$ e M3 e M2 $(\mathrm{p}=0,021)$. O eixo I do diagrama de ordenação (fig.1), que respondeu por 54,6\% da variação dos dados, está positivamente correlacionado com o grupo dos ácaros oribatídeos $(0,99)$, ou seja, quanto mais positivo o valor deste eixo, maior a abundância deste grupo. O eixo II, que respondeu por $25,0 \%$ da variação dos dados, está positivamente correlacionado com os 
Tabela I. Densidade média (indivíduos. $\mathrm{m}^{-2}$ ) e percentual $(\%)$ de diferentes grupos de microartrópodes do solo em sete fragmentos de mata com araucária no município de Muitos Capões, RS, Brasil, em junho de 2000.

\begin{tabular}{|c|c|c|c|c|c|c|c|c|c|c|}
\hline & \multicolumn{2}{|c|}{$\begin{array}{l}\text { Ácaros } \\
\text { Oribatídeos }\end{array}$} & \multicolumn{2}{|l|}{$\begin{array}{l}\text { Outros } \\
\text { Ácaros }\end{array}$} & \multicolumn{2}{|c|}{ Colêmbolos } & \multicolumn{2}{|c|}{$\begin{array}{l}\text { Outros } \\
\text { Artrópodes }\end{array}$} & \multicolumn{2}{|l|}{ Total } \\
\hline & ind. $m^{-2}$ & $\%$ & ind. $m^{-2}$ & $\%$ & ind. $\mathrm{m}^{-2}$ & $\%$ & ind. $m^{-2}$ & $\%$ & ind. $\mathrm{m}^{-2}$ & $\%$ \\
\hline A 1 & 51.015 & 59,4 & 22.617 & 26,3 & 7.080 & 8,2 & 5.231 & 6,1 & 85.943 & 100 \\
\hline A2 & 54.437 & 58,5 & 17.071 & 18,3 & 15.851 & 17,0 & 5.703 & 6,1 & 93.063 & 100 \\
\hline M1 & 52.082 & 58,9 & 22.214 & 25,1 & 11.461 & 13,0 & 2.655 & 3,0 & 88.412 & 100 \\
\hline M2 & 62.968 & 61,3 & 20.621 & 26,6 & 13.541 & 13,2 & 5.576 & 5,4 & 102.704 & 100 \\
\hline M3 & 38.114 & 47,8 & 21.201 & 25,5 & 15.537 & 19,5 & 4.838 & 6,1 & 79.689 & 100 \\
\hline M4 & 29.618 & 46,9 & 15.458 & 24,5 & 15.143 & 24,0 & 2.989 & 4,7 & 63.209 & 100 \\
\hline M5 & 46.728 & 57,9 & 20.311 & 25,1 & 10.355 & 12,8 & 3.363 & 4,2 & 80.756 & 100 \\
\hline
\end{tabular}

colêmbolos $(0,98)$. As parcelas dos fragmentos menores (M3, M4 e M5) têm menor abundância de oribatídeos (fig. 1). As parcelas de A1 e M5 mostram baixa abundância de colêmbolos, encontrando-se negativamente correlacionadas com o eixo II. A área M1 (mata ciliar), apesar de mostrar duas parcelas com uma baixa abundância de colêmbolos, apresentou em uma parcela a maior abundância deste grupo entre todas as áreas.

Pode-se perceber a diminuição na densidade de alguns grupos com a redução do tamanho dos capões de mato (fig. 2). Os pseudoescorpiões praticamente desaparecem nos menores. Entre os ácaros oribatídeos, deve-se considerar o fato de que principalmente nos fragmentos M2 e M5 uma espécie, Microppia cf. minus, foi amplamente dominante, de certa forma "mascarando" uma tendência de diminuição na abundância com a redução do tamanho dos fragmentos para a subordem Cryptostigmata. Exceção a esta tendência foram os miriápodes, representados principalmente pelas ordens Pauropoda e Symphyla, as quais tenderam a aumentar sua abundância nos fragmentos menores.

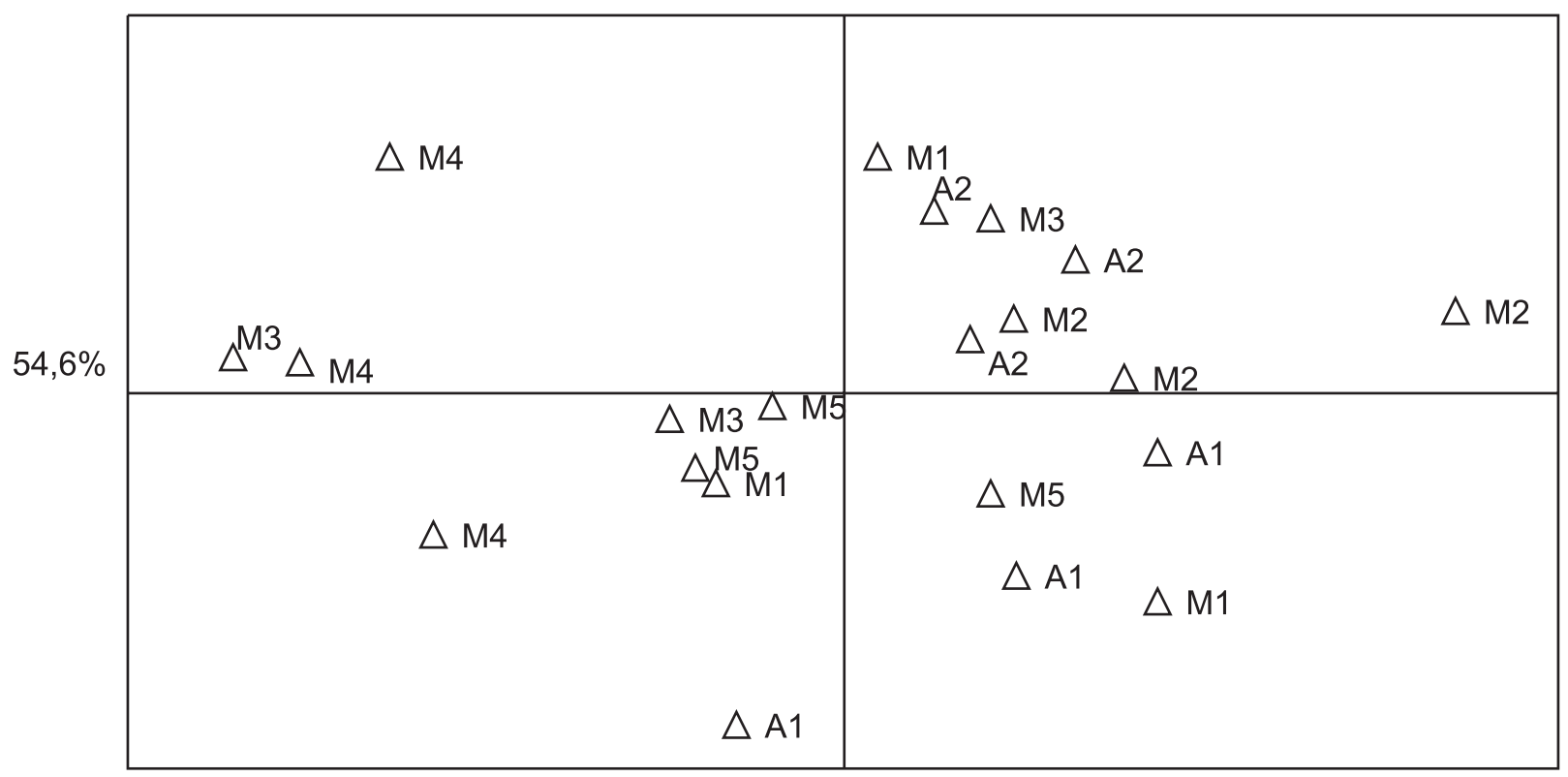

$25,0 \%$

Fig. 1. Diagrama de ordenação (coordenadas principais) de microartrópodes do solo em sete fragmentos de mata com araucária. O eixo 1 corresponde a $54,6 \%$ da variação dos dados e o eixo 2 representa $25 \%$ da variação dos dados. Descritores originais com coeficientes de correlação mais altos: eixo 1 , ácaros oribatídeos $(0,99)$; eixo 2, colêmbolos $(0,98)$. A1 e A2, fragmentos de mata com araucária da Estação Ecológica de Aracuri; M1-M5, fragmentos de mata com araucária da fazenda Boa Vista. Cada fragmento é representado por três parcelas de $10 \mathrm{~m}$ x $10 \mathrm{~m}$. Muitos Capões, RS, junho de 2000. 

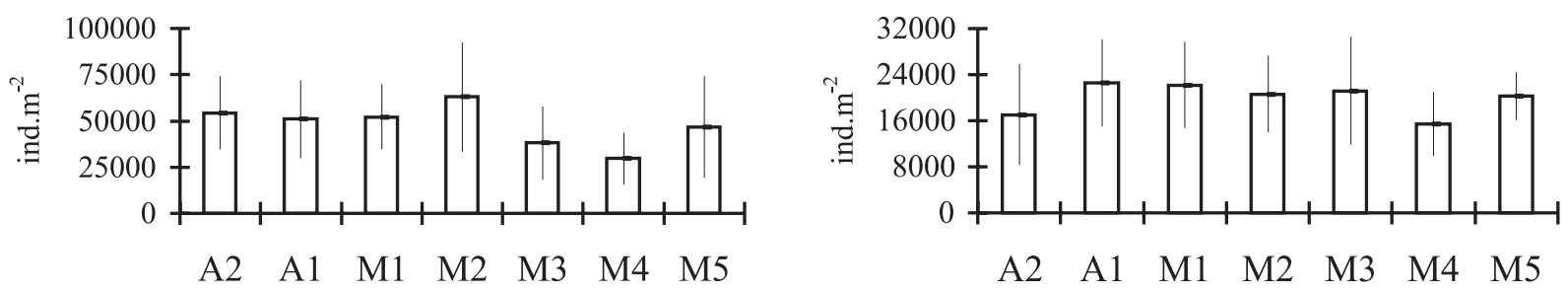

Collembola: Arthropleona

Collembola: Symphypleona
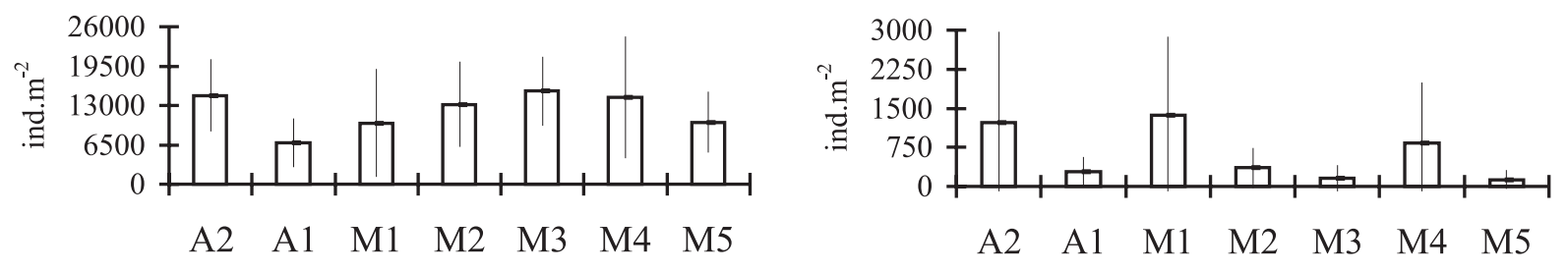

Araneae

Pseudoscorpiones
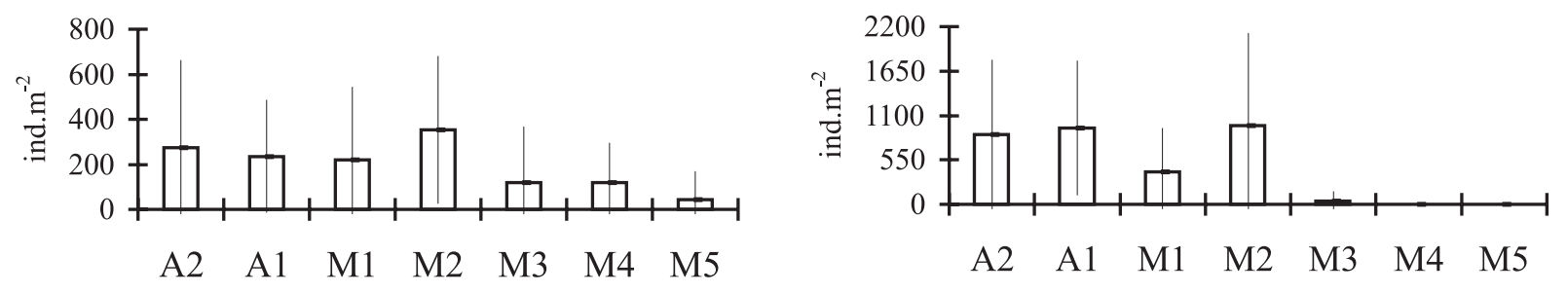

Coleoptera

Myriapoda
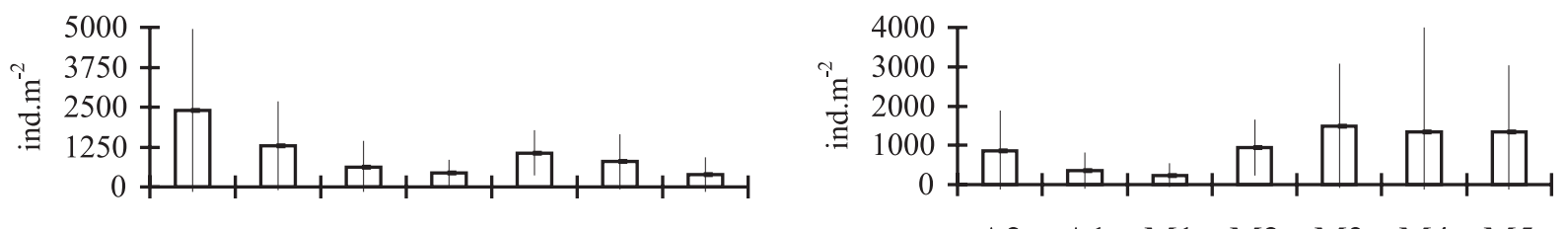

A2 A1 M1 M2 M3 M4 M5

A2 A1 M1 M2 M3 M4 M5

Fig. 2. Densidade média (indivíduos. $\mathrm{m}^{-2}$ ) dos principais grupos de microartrópodes encontrados nos seis centímetros superficiais (serapilheira+solo) de sete fragmentos de mata com araucária no município de Muitos Capões, RS, em junho de 2000 (A1 e A2, fragmentos de mata com araucária da Estação Ecológica de Aracuri; M1-M5, fragmentos de mata com araucária da fazenda Boa Vista). Colunas representam a densidade média e as barras verticais o desvio padrão. 


\section{DISCUSSÃO}

A qualidade do solo pode ser definida como a sua capacidade de funcionar dentro dos limites do ecossistema para sustentar a produtividade biológica, manter a qualidade do ambiente e promover a saúde de plantas e animais (KARLEn et al., 1997; DorAn \& ZEISS, 2000), sendo uma combinação das propriedades físicas, químicas e biológicas que contribuem para as funções do solo (KNOEPr et al., 2000). A saúde do solo é um conceito mais restrito, determinado principalmente por suas características ecológicas, como a diversidade biológica e a estabilidade (KARLEN et al., 1997; BRUGGEN \& Semenov, 2000). Ao longo das últimas décadas, muitos processos antropogênicos a curto e longo prazos vêm alterando em muito as características do solo e sua habilidade para suportar comunidades biológicas bem estruturadas, causando imensas perdas de biodiversidade (SIEPEL, 1996a) e afetando, consequentemente, a saúde do solo.

Muitos dos organismos e as comunidades como um todo, podem ser excelentes indicadores da qualidade e da saúde do solo de ecossistemas sujeitos a diferentes perturbações. RuF (1998) apresentou um índice de maturidade do solo baseado nas estratégias de vida de ácaros mesostigmatídeos em ecossistemas florestais da Alemanha e VAn StraAlen (1998) desenvolveu um sistema de bioindicação da acidez do solo a partir das preferências de $\mathrm{pH}$ de artrópodes nele presentes (colêmbolos, ácaros oribatídeos e opiliões).

Os ácaros oribatídeos têm geralmente pouca capacidade para responder a curto prazo às alterações ambientais; suas populações declinam rapidamente quando os hábitats são alterados, uma característica que pode permitir sua utilização para detectar a degradação ambiental (Behan-Pelletier, 1999; Siepel, 1996b). SGARDELIS \& Usher (1994), estudando a comunidade de ácaros oribatídeos em fragmentos florestais de áreas agrícolas da Inglaterra, relataram que as populações de oribatídeos e a organização da comunidade exibiram mudanças bruscas em uma transição entre mato-lavoura, com a maioria das espécies isoladas na área de mato e cercadas por um ambiente agrícola hostil. Hagvar apud Behan-Pelletier (1999) sugeriu que as mudanças ocorridas na estrutura de dominância de uma comunidade de microartrópodes do solo podem ser um pré-indicador de estresse. AOKI (1979) classificou os ácaros oribatídeos da família Oppiidae como "ambientalmente insensíveis", sendo geralmente comuns em hábitats perturbados. FRANCHINI \& RocketT (1996) relataram que Opiella nova (Oudemans, 1904) aumenta em número e em abundância relativa com o crescimento do impacto agrícola e estresse ambiental. Além dos opídeos, Aoki (1979) e BeHANPelletier (1999) destacaram como ambientalmente insensíveis as famílias Brachychtoniidae e Tectocepheidae, entre outras.

Os fragmentos menores e mais alterados da fazenda Boa Vista apresentaram alta dominância de opídeos, destacando-se Micropia cf. minus, juntamente com Tectocepheus cf. velatus (Tectocepheidae). De acordo com LAURANCE et al. (2002), ao longo do tempo, comunidades fragmentadas podem tornar-se crescentemente dominadas por generalistas que toleram os efeitos da matriz, por oportunistas adaptados aos distúrbios, e espécies com requisitos de área pequenos, como parece ser o caso entre os ácaros de muitas espécies de Oppiidae e Tectocepheidae (Aoki, 1979; Nakamura, 1989; Franchini \& Rockett, 1996; BeHAN-PELlETIER, 1999).

UsHer et al. (1993) estudaram a riqueza de espécies e a composição da fauna de coleópteros (Insecta) e aranhas (Arachnida) do solo em 28 remanescentes florestais na Inglaterra, encontrando uma correlação entre a riqueza de espécies e o processo de fragmentação florestal. Nos besouros, a riqueza relacionou-se mais com o formato da área, e nas aranhas com o grau de isolamento. No presente estudo, as aranhas e os pseudoescorpiões apresentaram diminuição drástica em sua abundância nos fragmentos menores, onde a estrutura da vegetação está mais alterada, a quantidade de serapilheira é menor e a pressão de pastoreio é maior. Segundo DenNis et al. (2001), a estrutura da vegetação, a quantidade de serapilheira e a pressão de pastoreio são importantes para a abundância e diversidade destes aracnídeos. GiLBert et al. (1998) e GonZALEZ \& CHANETON (2002) sugerem que, entre os microartrópodes do solo, os organismos predadores são fortemente influenciados pelo processo de fragmentação, necessitando de corredores ecológicos efetivos para manutenção de suas populações. Com relação aos coleópteros também ocorreu uma diminuição acentuada desde o fragmento maior e mais preservado (A2), até os menores.

Adis et al. (1987) relataram densidades de artrópodes do solo de cerca de 50000 ind. $\mathrm{m}^{-2}$ nos $14 \mathrm{~cm}$ superficiais do solo de uma floresta próxima de Manaus, com cerca de $77 \%$ ocorrendo nos três primeiros centímetros. As densidades encontradas no presente estudo são praticamente o dobro das obtidas por ADIs et al. (1987), com mais de $85 \%$ dos indivíduos ocorrendo nos três centímetros superficiais. As maiores densidades de microartrópodes do solo são encontradas geralmente em florestas de coníferas (PETERSEN \& LuXton, 1982; WALLWORK, 1983), com a maioria dos organismos ocorrendo nos três centímetros superficiais (UsHER, 1975). As densidades aqui obtidas podem ser consideradas de médias a altas quando comparadas com os estudos de ecossistemas florestais do mundo, compilados por Petersen \& Luxton (1982), embora alguns estudos apontem para densidades elevadíssimas em alguns ambientes (WALlwork, 1983; Migge et al., 1998).

A região de Muitos Capões, como o próprio nome indica, apresenta uma paisagem composta por uma matriz de campo recortada por uma infinidade de capões de mata com araucária. A ação antrópica vem afetando de forma radical a estrutura destes fragmentos, por meio da redução da área e até mesmo da eliminação de muitos destes nas últimas décadas.

Segundo Didham apud LAURANCE et al. (2002), o efeito-de-borda em fragmentos florestais na Amazônia pode alterar a abundância e biodiversidade dos invertebrados da serapilheira até uma distância de $100 \mathrm{~m}$. Caso esta informação também seja válida para as florestas com araucária, dos sete fragmentos analisados neste estudo, somente A1 e A2 apresentam um ambiente de interior de floresta relativamente preservado. 
A maioria dos fragmentos florestais no mundo está de maneira geral nas mãos de proprietários privados, sendo que o futuro dessas áreas e da biodiversidade a elas associada depende em muito da atitude das comunidades locais (BAUNDRY, 1993), pois grande parte da biodiversidade ainda existente em paisagens intensamente cultivadas está localizada nestes fragmentos, cuja dinâmica é pouco conhecida.

Remanescentes florestais podem ser considerados, em paisagens essencialmente agrícolas, pontos críticos ("hot spots") para os organismos do solo (HAGVAR, 1998), sendo muitas vezes os últimos refúgios existentes para a fauna em geral (CHIARELlo, 2000). Os resultados aqui obtidos, ainda que preliminares, sugerem que os fragmentos pequenos e mais alterados de mata com araucária sofrem uma redução acentuada na abundância de diversos grupos da fauna do solo, principalmente os ácaros oribatídeos, os coleópteros e os pseudoescorpiões e, paralelamente, um aumento da abundância de grupos mais tolerantes aos efeitos da fragmentação e pastoreio.

Esta diminuição na abundância de microartrópodes e também da diversidade de oribatídeos, uma vez que nos fragmentos menores tende a ocorrer uma alta dominância de uma espécie (Microppia cf. minus), pode comprometer, a médio e longo prazos, os processos de decomposição e mineralização da matéria orgânica, afetando a qualidade e a saúde do solo (KARLEN et al., 1997; DORAN \& ZEISS, 2000) e conseqüentemente todo o sistema ecológico, uma vez que os solos não são meros componentes, mas sim controladores de diversos processos que garantem o funcionamento dos ecossistemas terrestres (PAUL, 1989).

Agradecimentos. À FAPERGS e ao CNPq pela bolsa de Recém-doutor desenvolvida no Depto. Ecologia/UFRGS. À Dra. Sandra M. Hartz, Enga. Simone Winck, Matheus Duarte, Patrick Colombo, Jose Cerveira e Marcelo Saraiva (UFRGS), pelo auxílio durante as amostragens. Ao IBAMA-RS e ao Sr. Elcino Duarte Borges, proprietário da fazenda Boa Vista, pelo acesso às áreas de amostragem.

\section{REFERÊNCIAS BIBLIOGRÁFICAS}

Adis, J.; Morais, J. W. \& Ribeiro, E. F. 1987. Vertical distribution and abundance of arthropods in the soil of a neotropical secondary forest during the dry season. Tropical Ecology, Varanasi, 28:174-181.

Aoki, J. 1979. Difference in sensitivities of oribatid families to environmental change by human impacts. Revue d'Écologie et de Biologie du Sol, Paris, 16:415-422.

Baker, E. W. \& Wharton, G. W. 1959. An introduction to Acarology. New York, McMillan. 465p.

Balogh, J. 1972. Oribatid genera of the World. Budapeste, Akadémiai Kiado. 330p.

Balogh, J. \& Balogh, P. 1988. Oribatids of the Neotropical Region. Amsterdam, Elselvier. v.1. 335p.

1990. Oribatids of the Neotropical Region. Amsterdam, Elselvier. v. 2. 333p.

BAUNDRY, J. 1993. Interactions between agricultural and ecological systems at the landscape level. Agriculture, Ecosystem and Environment, Amsterdam, 27:119-130.

Beare, M. H.; Coleman, D. C. et al. 1995. A hierarchical approach to evaluating the significance of soil biodiversity to biogeochemical cycling. Plant and Soil, The Hague, 170:5-22.

Behan-Pelletier, V. M. 1999. Oribatid mite biodiversity in agroecosystems: role for bioindication. Agriculture,
Ecosystem and Environment, Amsterdam, 74:411-423. Behling, H. 1997. Late quaternary vegetation, climate and fire history in the Araucaria forest and campos region from Serra Campos Gerais (Paraná), south Brazil. Review of Palaeobotany and Palynology, Amsterdam, 97:109121.

BrAsil, 1993. Recursos naturais e meio ambiente, uma visão do Brasil. Rio de Janeiro, IBGE. 154p.

Bruggen, A. H. C. \& Semenov, A. M. 2000. In search of biological indicators of soil health an disease suppression. Applied Soil Ecology, Amsterdam, 15:13-24.

Butcher, J. W.; Snider, R. \& Snider, R. J. 1971. Bioecology of edaphic Collembola and Acarina. Annual Review of Entomology, Palo Alto, 16:249-288.

Cabrera, A. L. \& Willink, A. 1980 . Biogeografia de America Latina. 2. ed. Washington, Departamento de Assuntos Científicos, O.E.A. 121p.

Cancela da Fonseca, J. P. 1990. Forest management: impact on soil microarthropods and soil microorganims. Revue d'Écologie et de Biologie du Sol, Paris, 27(3):269-283.

Cestaro, L. A.; Waechter, J. L. \& Batista, L. R. M. 1986. Fitossociologia do estrato herbáceo da mata de Araucária da Estação Ecológica de Aracuri, Esmeralda, RS. Hoehnea, São Paulo, 13:59-72.

Chiarello, A. G. 2000. Conservation value of a native forest fragment in a region of extensive agriculture. Revista Brasileira de Biologia, Rio de Janeiro, 60(2):237-247.

Culik, M. P.; Souza, J. L. \& Ventura, J. A. 2002. Biodiversity of Collembola in tropical agricultural environments of Espírito Santo, Brasil. Applied Soil Ecology, Amsterdam, 21(1):49-58.

Dennis, P.; Young, M. R. \& Bentley, C. 2001. The effects of varied grazing management on epigeal spiders, harvestmen and pseudoscorpions of Nardus stricta grassland in upland Scotland. Agriculture, Ecosystem and Environment, Amsterdam, 86:39-57.

DindAL, D. L. 1990. Soil biology guide. New York, John Wiley \& Sons. 1349p.

Donegan, K. K.; Watrud, L. S. et al. 2001. Soil and litter organims in Pacific northwest forests under different management practices. Applied Soil Ecology, Amsterdam, 18:159-175.

Doran, J. W. \& Zeiss, M. R. 2000. Soil health and sustainability: managing the biotic component of soil quality. Applied Soil Ecology, Amsterdam, 15:3-11.

Duarte, M. M. \& Becker, M. 2000. A comunidade de microartrópodes em solos da micro-região carbonífera do baixo rio Jacuí. In: Carvão e meio ambiente. Porto Alegre, Ed. da Universidade. p.695-725.

Eisenbeis, G. \& Whichard, W. 1987. Atlas on the biology of soil arthropods. Berlin, Springer-Verlag. 437p.

Fleig, M. 1990. Líquens da Estação Ecológica de Aracuri. Novas ocorrências no Rio Grande do Sul. Iheringia, Sér. Bot., Porto Alegre, (40):121-125.

Franchini, P. \& Rockett, C. L. 1996. Oribatid mites as "indicator" species for estimating the environmental impact of conventional and conservation tillage practices. Pedobiologia, Jena, 40:217-225.

Franklin, E. N.; Woas, S. et al. 1998. Ácaros oribatídeos (Acari: Oribatida) arborícolas de duas florestas inundáveis da Amazônia central. Revista Brasileira de Biologia, Rio de Janeiro, 58(2):317-335.

Gilbert, F.; Gonzalez, A. \& Evans-Freke, I. 1998. Corridors maintain species richness in the fragmented landscapes of a microecosystem. Proceedings of the Royal Society of London, London, 265:577-582.

Gonzalez, A. \& Chaneton, E. J. 2002. Heterotroph species extinction, abundance and biomass dynamics in an experimentally fragmented microecosystem. Journal of Animal Ecology, Oxford, 71:594-602.

Hagvar, S. 1998. The relevance of the Rio-Convention on biodiversity to conserving the biodiversity of soils. Applied Soil Ecology, Amsterdam, 9:1-7.

Harada, A. Y. \& Bandeira, A. G. 1994a. Estratificação e densidade de invertebrados em solo arenoso sob floresta primária e plantios arbóreos na Amazônia central durante 
a estação seca. Acta Amazonica, Manaus, 24(1/2):103118

1994b. Abundância e estratificação de invertebrados em solo argiloso sob floresta e plantios arbóreos na Amazônia central, durante a estação seca. Boletim do Museu Paraense Emílio Goeldi, Série Zoologica, Belém, 10(2):235-250.

Heneghan, L. \& Bolger, T. 1998. Soil microarthropod contribution to forest ecosystem processes: the importance of observational scale. Plant and Soil, The Hague, 205(2): $113-124$.

Karlen, D. L.; Mausbach, M. J. et al. 1997. Soil quality: a concept, definition and framework for evaluation. Soil Science Society of American Journal, Madison, 61:410 .

Knoepr, J. D.; Coleman, D. C. et al. 2000. Biological indices of soil quality: an ecosystem case study of their use. Forest Ecology and Management, Amsterdam, 138:357-368.

Laurance, W. F.; Lovejoy, T. E. et al. 2002. Ecosystem decay of Amazonian forest fragments: a 22-year investigation. Conservation Biology, Boston, 13(3):605-618.

Lavelle, P.; Lattaud, C. et al. 1995. Mutualism and biodiversity in soils. Plant and Soil, The Hague, 170:23-33.

Longhi-Wagner, H. M. \& Boldrini, I. I. 1988. Gramíneas da Estação Ecológica de Aracuri, Esmeralda, Rio Grande do Sul. Iheringia, Sér. Bot., Porto Alegre, (38):21-42.

Maraun, M.; Visser, S. \& Scheu, S. 1998. Oribatid mites enhance the recovery of the microbial community after a strong disturbance. Applied Soil Ecology, Amsterdam, 9: $175-181$

Migge, S.; Maraun, M. et al. 1998. The oribatid mite community (Acarina) of pure and mixed stands of beech (Fagus sylvatica) and spruce (Picea abies) of different ages. Applied Soil Ecology, Amsterdam, 9:115-121.

Mueller, B. R.; Beare, M. H. \& Crossley, D. A., JR. 1990. Soil mites in detrital food webs of conventional and no-tillage agroecosystems. Pedobiologia, Jena, 34:389-401.

NAKAmura, Y. 1989. Oribatids and enchytraeids in ecofarmed and conventionally farmed dryland grainfields of central Japan. Pedobiologia, Jena, 33:389-398.

Paul, E. A. 1989. Soils as components and controllers of ecosystem processes. In: Grubb, P. J. \& Whittaker, J. B. eds. Toward a more exact ecology. Oxford, Blackwell. p. 353-374.

Petersen, H. \& Luxton, M. 1982. A comparative analysis of soil fauna populations and their role in decomposition processes. Oikos, Copenhagen, 39(3):287-388.

Pillar, V. DE P. \& Orlóci, L. 1996. On randomization testing in vegetation science: multifactor comparisons of relevé groups. Journal of Vegetation Science, Uppsala, 7:585592

Ribeiro, E. F. \& Schubart, H. O. R. 1989. Oribatídeos (Acari: Oribatida) colonizadores de folhas em decomposição de três sítios florestais da Amazônia central. Boletim do Museu Paraense Emílio Goeldi, Sér. Zool., Belém, 5(2):243-276.

RuF, A. 1998. A maturity index for predatory soil mites (Mesostigmata: Gamasida) as an indicator of environmental impacts of pollution on forest soils. Applied Soil Ecology, Amsterdam, 9:447-452.

Rusek, J. \& Marshall, V. G. 2000. Impact of airborne pollutants on soil fauna. Annual Review of Ecology and Systematics, Palo Alto, 31:395-423.

Saunders, D. A.; Hobbs, R. J. \& Margules, C. R. 1991. Biological consequences of ecosystem fragmentation: a review. Conservation Biology, Boston, 5(1):18-52.

Seadsted, T. R. 1984. The role of microarthropods in decomposition and mineralization process. Annual Review of Entomology, Palo Alto, 29:25-46.

Sgardelis, S. P. \& Usher, M. B. 1994. Responses of soil Cryptostigmata across the boundary between a farm woodland and an arable field. Pedobiologia, Jena, 38:3649.

SiePel, H. 1996a. Biodiversity of soil microarthropods: the filtering of species. Biodiversity Conservation, Amsterdam, 5:251-260.

1996b. The importance of unpredictable and short-term environmental extremes for biodiversity in oribatid mites. Biodiversity Letters, Oxford, 3:26-34.

Silva, G. M. DA. 1996. O desenvolvimento sustentado na região do domínio da floresta com Araucária. In: Alvarez V. V. H.; Fontes, L. E. F. \& Fontes, M. P. F. eds. O solo nos grandes domínios morfoclimáticos do Brasil e o desenvolvimento sustentado. Viçosa, Sociedade Brasileira de Ciência do Solo, Universidade Federal Viçosa. p. 217-232.

Usher, M. B. 1975. Seasonal and vertical distribution of a population of soil arthropods: Cryptostigmata. Pedobiologia, Jena, 15:364-374.

Usher, M. B.; Field, J. P. \& Bedford, S. E. 1993. Biogeography and diversity of ground-dwelling arthropods in farm woodlands. Biodiversity Letters, Oxford, 1:54-62.

Ushiwata, C. T.; Sautter, K. D. \& Kobiyama, M. 1995 Influence of compactation of a forest soil on the soil fauna in a subtropical region. I. Oribatei (Acari: Cryptostigmata) and Collembola (Insecta). Revista Brasileira de Zoologia, Curitiba, 12(4):905-913.

Vallejo, L. R.; Fonseca, C. L. Da \& Gonçalves, D. R. P. 1987. Estudo comparativo da mesofauna do solo entre áreas de Eucaliptus citriodora e mata secundária heterogênea. Revista Brasileira de Biologia, Rio de Janeiro, 47(3):363-370.

Van Straalen, N. 1998. Evaluation of bioindicator systems derived from soil arthropod communities. Applied Soil Ecology, Amsterdam, 9:429-437.

Van Straalen, N.; Kraak, M. H. S. \& Denneman, C. A. J. 1988 Soil microarthropods as indicators of soil acidification and decline in the Veluwe area, the Netherlands. Pedobiologia, Jena, 32:47-55.

Waechter, J. L.; Cestaro, L. A. \& Miotto, S. T. S. 1984 Vegetation types in the Ecological Station of Aracuri, Esmeralda, Rio Grande do Sul, Brazil. Phytocoenologia, Stuttgart, 12(2/3):261-269.

Wallwork, J. A. 1970. Ecology of soil animals. London, MacGraw-Hill. 283p.

1983. Oribatids in forest ecosystems. Annual Review of Entomology, Palo Alto, 28:109-130.

Wardle, D. A. \& Giller, K. E. 1996. The quest for a contemporary ecological dimension of soil biology. Soil Biology and Biochemistry, Oxford, 28(11):1549-1554.

Wardle, D. A.; Yeates, G. W. et al. 1995. The detritus foodweb and the diversity of soil fauna as indicators of disturbance regimes in agroecosystems. Plant and Soil, The Hague, 170:35-43.

Whitford, W. G. 1996. The importance of the biodiversity of soil biota in arid ecosystems. Biodiversity Conservation, London, 5:185-195.

WitKamp, P. 1971. Soils as components of ecosystems. Annual Review of Ecology and Systematics, Palo Alto, 2:85-110. 